\title{
Resonant photoelectron diffraction with circularly polarized light
}

\author{
Martin Morscher, ${ }^{1}$ Frithjof Nolting, ${ }^{2}$ Thomas Brugger, ${ }^{1}$ and Thomas Greber ${ }^{1, *}$ \\ ${ }^{1}$ Physik-Institut, Universität Zürich, Winterthurerstrasse 190, CH-8057 Zürich, Switzerland \\ ${ }^{2}$ Paul Scherrer Institut, $\mathrm{CH}-5232$ Villigen PSI, Switzerland \\ (Received 8 September 2011; published 6 October 2011)
}

\begin{abstract}
The atomic and magnetic structure of surfaces and interfaces can be determined by angle-scanned resonant x-ray photoelectron diffraction. For the case of magnetized nickel the resonant $L_{2}$ excitation with circularly polarized light yields electrons with a dichroic signature from which the dipolar part may be retrieved. The dichroic dipoles in the electron emission patterns rotate by distinct angles, which indicates different electron source waves with angular momenta parallel or antiparallel to those of the photons. For precise determination of the magnetization direction, the asymmetry between electron diffraction patterns as excited by left and right circularly polarized light has to be used.
\end{abstract}

DOI: 10.1103/PhysRevB.84.140406

PACS number(s): 75.25.-j, 61.05.js, 79.60.Bm

The quest for atomic-scale structure determination at surfaces and interfaces has lead to the development of a large number of powerful methods. ${ }^{1}$ Among those, x-ray photoelectron spectroscopy with angular resolution (XPD) allows structure determination paired with chemical and magnetic sensitivity. ${ }^{2}$ The signal is best when the $\mathrm{x}$-ray absorption coefficient is at maximum. These maxima occur in resonant excitation and have so far been exploited for the probing of defect states in $\mathrm{TiO}_{2},{ }^{3}$ for looking inside an endofullerene, ${ }^{4}$ or for the investigation of the mixed valence structure of magnetite. ${ }^{5}$

In this Rapid Communication, resonant photoelectron diffraction (RXPD) is applied to nickel, a prototype system of resonant photoemission. ${ }^{6-10}$ Circularly polarized light is used for precise measurement of the X-ray magnetic circular dichroism, which is largest at resonance. The angle-scanned experiment allows the reconstruction of a dichroic dipole. If only one light helicity is used, the angular momentum of the electron source wave ${ }^{11,12}$ rotates the dichroic dipole by small angles away from the nominal magnetization direction. This extends the results of Daimon et al., who demonstrated that forward scattering patterns rotate due to the angular momentum of photons. ${ }^{13-15}$ For the present case it is found that Auger electrons may carry angular momenta larger than $\hbar$ and opposite to that of the exciting photon.

Circular magnetic dichroism $\Delta I_{\mathrm{MD}}$ is the difference between the absorption coefficient of right and left circularly polarized light. It is proportional to the scalar product of the magnetization $\mathbf{m}$ and the angular momentum of the incoming photon $\mathbf{L}_{\mathrm{ph}}:{ }^{16}$

$$
\Delta I_{\mathrm{MD}} \propto \mathbf{m} \cdot \mathbf{L}_{\mathrm{ph}} \cdot
$$

For right circularly polarized light $\left(\sigma^{+}\right) \mathbf{L}_{\mathrm{ph}}$ is parallel to the propagation direction of the photon, and for left circularly polarized light $\left(\sigma^{-}\right) \mathbf{L}_{\mathrm{ph}}$ is antiparallel. $\Delta I_{\mathrm{MD}}$ is proportional to the cosine of the angle between $\mathbf{m}$ and $\mathbf{L}_{\mathrm{ph}}$ and from this the absolute orientation of $\mathbf{m}$ can be determined from three or more noncoplanar light incidences.

Often $\Delta I_{\mathrm{MD}}$ is determined from the difference of the total photoelectron yield. This must not hold for partial measurements as in angular-resolved photoemission. ${ }^{17}$ The different photoelectron source waves caused by differently polarized light lead to different final states that comprise information on the magnetism and the surrounding of the emitter. ${ }^{18}$ In our experiment, where $\mathbf{m}$ and $\mathbf{L}_{\mathrm{ph}}$ are rotated with respect to each other, $\Delta I_{\mathrm{MD}}$ displays as a (dichroic) dipole, which may be easily distinguished from higher-order multipoles that contain structural information.

The experiments have been performed at the SIM beamline at the Swiss Light Source (SLS) ${ }^{19}$ in an endstation dedicated for angle-resolved $\mathrm{x}$-ray photoelectron diffraction with a base pressure below $2 \times 10^{-10}$ mbar. The geometry of the experiment is sketched in Fig. 1. All measurements were done at room temperature. The $\mathrm{Ni}(111)$ yoke crystal $^{20}$ was cleaned by repeated cycles of argon sputtering and annealing. It is magnetized by passing a current of $2 \mathrm{~A}$ for $30 \mathrm{~s}$ through the yoke coil. The resulting magnetization was inferred from $\mathrm{x}$-ray magnetic circular dichroism (XMCD), and a comparison with data of Chen et al. ${ }^{21}$ indicates a magnetization of $\sim 40 \%$, which is not $100 \%$ due to a multidomain structure.

Resonant photoemission on $3 d$ transition metals is most intense at the $L_{3}$ absorption edge. ${ }^{3,21}$ Here we investigate the $L_{2}$ resonance since it provides $L_{2} M M$ and $L_{3} M M$ emission, which allows for direct comparison and consistency checks. Figure 2 shows $x$-ray photoelectron spectra from magnetized $\mathrm{Ni}(111)$ of right and left circularly polarized light. The photon energy is set on the Ni $L_{2}$ resonance $\left(2 p_{1 / 2} \rightarrow 3 d\right)$. The Fermi level at $870.5 \mathrm{eV}$, the $L_{2} M M(863.8 \mathrm{eV}) 6 \mathrm{eV}$ satellite (see Ref. 22 and references therein), and the $L_{3} M M(846.2 \mathrm{eV})$ Auger deexcitation peak are most prominent. The spectra have been normalized with the photon flux. Figure 2(b) demonstrates circular dichroism in these electron emission spectra. The asymmetry $A=\left[I\left(\sigma^{+}\right)-I\left(\sigma^{-}\right)\right] /\left[I\left(\sigma^{+}\right)+I\left(\sigma^{-}\right)\right]$ between right and left circularly polarized light exhibits a maximum at $\alpha_{2}$ and a minimum at $\alpha_{3}$. The asymmetry can be reversed by switching the magnetization or by the rotation of the sample by $180^{\circ} .{ }^{23}$ Off resonance, at $\hbar \omega=873.5 \mathrm{eV}$, the dichroic asymmetry in the $L_{2} M M$ Auger line is $1.4 \pm 0.8 \%$ (data not shown). ${ }^{24}$ The extrema $\alpha_{2}$ and $\alpha_{3}$ do not exactly coincide with the $L_{2} M M$ and the $L_{3} M M$ intensity maxima, which indicates a multiplet structure. ${ }^{25}$ In the following we use the labels $\alpha_{i}^{\sigma}$ for electrons at the energies of $\alpha_{2}$ and $\alpha_{3}$, excited with $\sigma^{+}$and $\sigma^{-}$polarized radiation, respectively.

If we perform angle-scanned x-ray photoelectron diffraction with circularly polarized light on the $L_{2}$ resonance, the experiment yields information on the atomic and the 


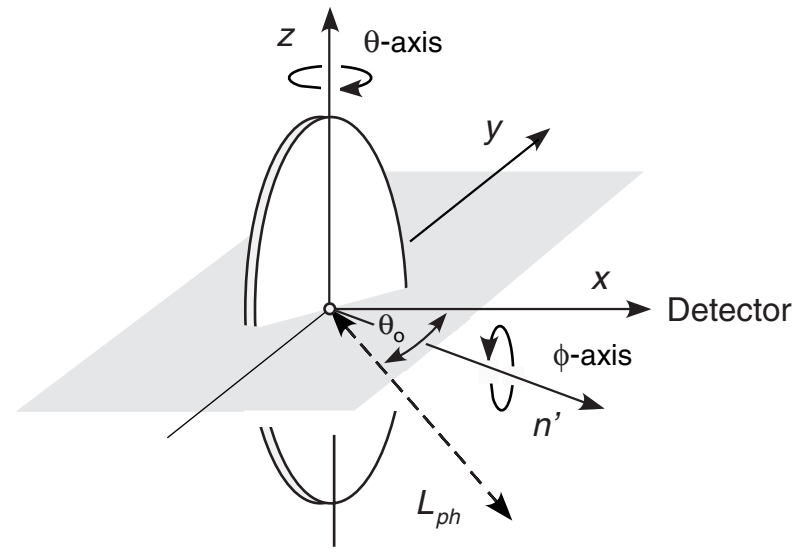

FIG. 1. Geometry of the XPD experiment. The electron detection is parallel to $\mathbf{x}$ and the polar $(\theta)$ rotation axis parallel to $\mathbf{z}$. The sample normal $\mathbf{n}^{\prime}$ is the azimuthal $(\phi)$ rotation axis and lies with the light incidence along its angular momentum $\mathbf{L}_{\mathrm{ph}}$ in the $x y$ plane (shaded area), $\theta_{o}=55^{\circ}$ away from $\mathbf{x}$.

magnetic structure. The photoelectron intensity $I$ is mapped in polar coordinates $[f(\theta), \phi]$, where the polar angle $\theta$ and the azimuthal angle $\phi$ define the sample orientation with respect to the electron detection direction (see Fig. 1). ${ }^{26}$ This leads for $\Delta I_{\mathrm{MD}}$ to a dipolar function $D(\theta, \phi)$ in the XPD

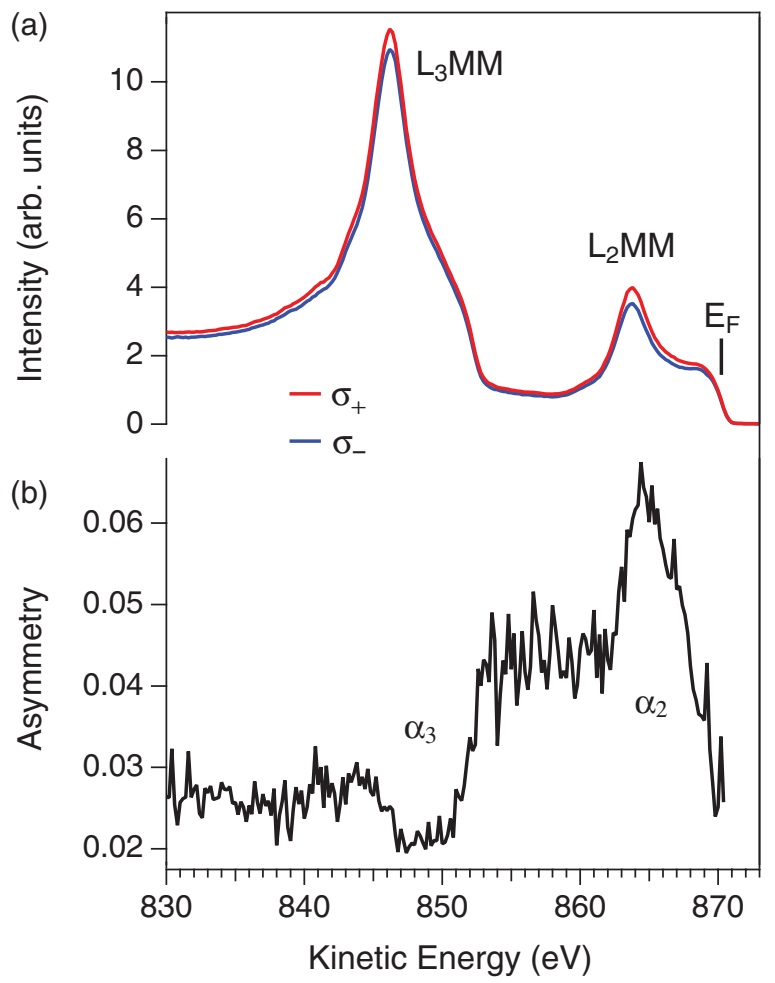

FIG. 2. (Color online) (a) Near normal photoelectron spectra with left and right circularly polarized light with an angle of $30^{\circ}$ and $180^{\circ}-30^{\circ}$ between $\mathbf{L}_{\mathrm{ph}}$ and $\mathbf{m}^{\prime}$. The photon energy is set on the Ni $L_{2}$ resonance at $\hbar \omega=870.5 \mathrm{eV}$. The spectra have been normalized with the photon flux. The Fermi level $E_{F}$, and the $L_{2} M M$ and the $L_{3} M M$ Auger deexcitation peaks are indicated. (b) The asymmetry between right and left circularly polarized light exhibits two distinct extrema $\alpha_{2}$ and $\alpha_{3}$ on which we performed XPD measurements. (a)

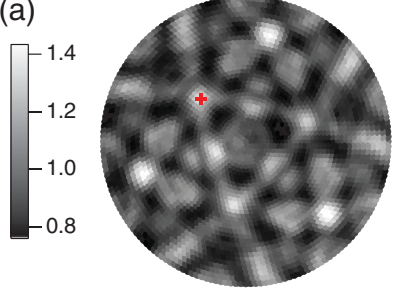

(c)
-0.05
-0.00
-0.05

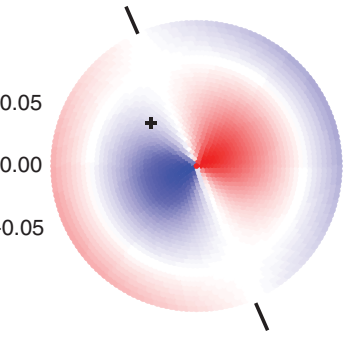

(b)

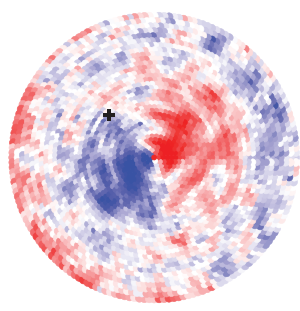

(d)

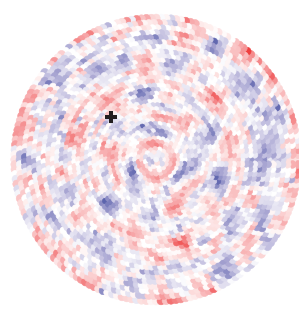

FIG. 3. (Color online) (a) Resonant $x$-ray photoelectron diffraction (RXPD) data of $\mathrm{Ni}(111)$. The 3500 stereographically projected data points for $\alpha_{2}^{+}$cover polar angles $0 \leqslant \theta \leqslant 70^{\circ}$. The data are $\phi$ averaged, i.e., normalized at each polar angle with the corresponding average intensity. (b) Asymmetry of two XPD data sets at $\alpha_{2}$ measured with right and left circularly polarized light. The twofold, i.e., dipolar pattern reveals the direction of the magnetization. (c) $D$-function fit to the asymmetry in (b). The direction of magnetization $\mathbf{m}^{\prime}$ and ticks for the corresponding azimuthal orientation at $\phi_{m} \pm 90^{\circ}$ are indicated. The [011] direction (cross) and the yoke axis along [11̄0] are marked. (d) Residuum of the fit in (c) with respect to (b).

map that depends on $\mathbf{L}_{\mathrm{ph}}$, the electron detection direction in the laboratory frame and $\mathbf{m}^{\prime}\left(\theta_{m}, \phi_{m}, a_{m}\right)$ in the sample frame, where the amplitude $a_{m}$ is a measure for the magnitude of the dichroism. ${ }^{23}$

Figure 3(a) shows data for $\alpha_{2}^{+}$. The XPD map is dominated by the information on the atomic structure which corresponds to that of a face-centered-cubic (fcc) crystal which is cut along the (111) plane. ${ }^{27}$ Below the obvious atomic structure, dichroic information must be hidden. In order to visualize the dichroism, we form the asymmetry $A$ between the $\alpha_{2}^{+}$ and the $\alpha_{2}^{-}$XPD scans [see Fig. 3(b)]. These data contain information on the dipolar (magnetic) part of the dichroism and higher-order multipoles, where the latter are related to differences in the diffraction patterns due to the forward scattering peak rotation. ${ }^{13,28}$ The dipolar part has a symmetry as it is expected for in-plane magnetization. Figure 3(c) shows the fit of a dipolar function $D(\theta, \phi)$, which determines $\mathbf{m}^{\prime}$. We find $\theta_{m}=89.0 \pm 1^{\circ}$, and $\phi_{m}=39.1 \pm 1^{\circ}$, where $\phi=0$ is set to the [110] direction. This result is consistent with spin-polarized photoemission. ${ }^{20}$ The rotation of the sample imposes on $D$ two nodal lines $\left(\mathbf{m}^{\prime} \cdot \mathbf{L}_{\mathrm{ph}}=0\right)$ : a circle at $\theta_{o}=55^{\circ}$ and a diameter perpendicular to $\phi_{m}$. In Fig. 3(d) the residuum of the asymmetry and $D$ is shown. It has the $C_{3}$ symmetry of the substrate and indicates further differences in the diffraction patterns due to different source waves created by the $\sigma^{+}$and $\sigma^{-}$photons, respectively. Such effects have been pioneered by Daimon et al., where they showed that the angular momenta of the photons are transferred to the photoelectrons, which in turn lead to an emitter scatterer distance-dependent rotation of the forward scattering peak. ${ }^{13,14}$ 
The orientation of the dipole in Fig. 3(b) must represent the magnetization direction because rotation effects due to light angular momenta cancel in this asymmetry. As shown in the following, this changes for an individual XPD scan with either $\sigma^{+}$or $\sigma^{-}$radiation. If we fit $D$ to the data in Fig. 3(a), or the ones recorded with $\sigma^{-}$polarization, we find dichroic dipoles which are within $\pm 6^{\circ}$, consistent with the magnetization direction as found from Fig. 3(b). Although this has the practical advantage that the magnetization can be inferred without switching the light polarization, it is not very accurate since the $D$ function is much weaker than the forward-scattering-induced XPD patterns.

If we want to extract more quantitative information on the rotation of the $D$ functions upon use of photons with plus or minus $\hbar$ angular momentum we have to perform a normalization that removes the forward scattering intensity modulations but preserves the angular momentum of $\sigma^{+}$or $\sigma^{-}$. We do so in using the $\phi$-averaged data $\bar{\alpha}_{i}^{\sigma}$ and form $\Delta \alpha_{2}^{\sigma}=2 \bar{\alpha}_{2}^{\sigma} /\left(\bar{\alpha}_{3}^{+}+\bar{\alpha}_{3}^{-}\right)-1$ and vice versa.

As $\alpha_{2}$ and $\alpha_{3}$ electrons are expected to have very similar XPD patterns ${ }^{12}$ - the wavelength difference is $1 \%$-most XPD information on the atomic structure should be canceled, though the $\Delta \alpha_{i}^{\sigma}$ may show a polarization-dependent rotation of the dichroic dipole. For $\alpha_{2}$, we find a rotation $\Delta \phi_{m}$ of $\pm 4.2^{\circ}$ around the value of Fig. 3(b). This has the same origin as the forward scattering peak rotation. ${ }^{13}$ Essentially, the angular momentum of an outgoing photoelectron induces a rotation of all features in the XPD patterns with respect to the crystal lattice. For single scattering the maximum angle of rotation $\gamma_{\max }$ is given by $n \hbar /\left[R p \sin ^{2}\left(\theta_{o}\right)\right]$, where $R$ is the distance between emitter and scatterer, $p$ the momentum of the outgoing electron, and $\theta_{o}$ the angle between the light incidence and the electron detection (see Fig. 1). ${ }^{14,29}$ For nickel, $n=1$, an electron kinetic energy of $850 \mathrm{eV}$, and $\theta_{o}=55^{\circ}, \gamma_{\max }$ gets $2.2^{\circ}$. Of course, the angular shift is not isotropic, because it depends on the angle between the electron angular momentum and the nearest-neighbor directions. However, for a fcc material, as is nickel, the 12 nearest neighbors of an emitter in the bulk sit on a sphere with radius $R$, on the vertices of a cuboctahedron and must lead to a fairly isotropic rotation of the XPD patterns around the axis of the incoming photons. The $\Delta \phi_{m}\left(\alpha_{2}\right)$ 's have the same sense of rotation as the corresponding photon angular momentum and are compatible with the transfer of $2 \hbar$ angular momentum to the emitted electrons. This result does not correspond to the transfer of a single $\hbar$ to the emitted electron, as it is expected for direct photoelectron emission, ${ }^{13}$ and it signals correlations between the $2 p_{1 / 2}$ hole and the $3 d$ shell.

Figure 4 shows the $\Delta \alpha_{2}^{\sigma}$ and the $\Delta \alpha_{3}^{\sigma}$ XPD scans for $\sigma^{+}$and $\sigma^{-}$radiation. Dipoles as in Fig. 3(b) appear where the sign changes upon change in the polarization. The $D$ functions for $\Delta \alpha_{2}^{ \pm}$and $\Delta \alpha_{3}^{ \pm}$display $\Delta \phi_{m}$ 's of $\pm 4.2^{\circ}$ and $\mp 12.6^{\circ}$, respectively. We want to note that the use of more than 3000 different photon incidence angles allows a very accurate $\pm 0.8^{\circ}$ determination of the $\Delta \phi_{m}\left(\alpha_{2}\right)$ 's and permits for single quantum assignments $2 \hbar(-2 \hbar)$. In the $\Delta \alpha_{3}$ patterns with a lower asymmetry (see Fig. 2) the error increases by a factor of 3 and makes it compatible with angular momenta of $-6 \pm 2 \hbar$ or $6 \pm 2 \hbar$ [see Fig. 4(e)]. This surprising result implies that $L_{3} M M$ Auger electron channels may produce electrons with
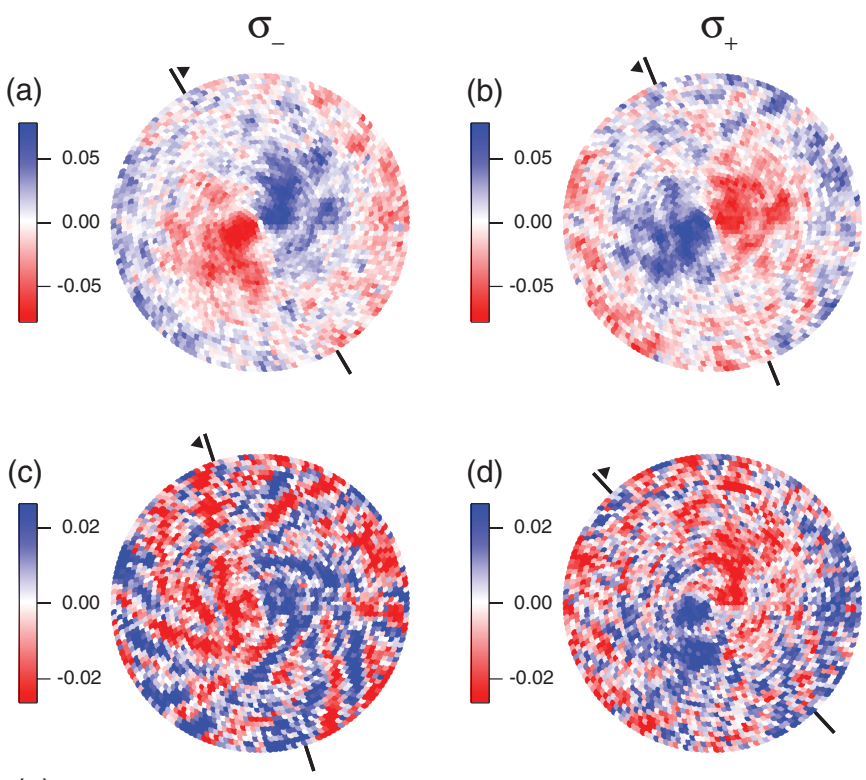

(e)

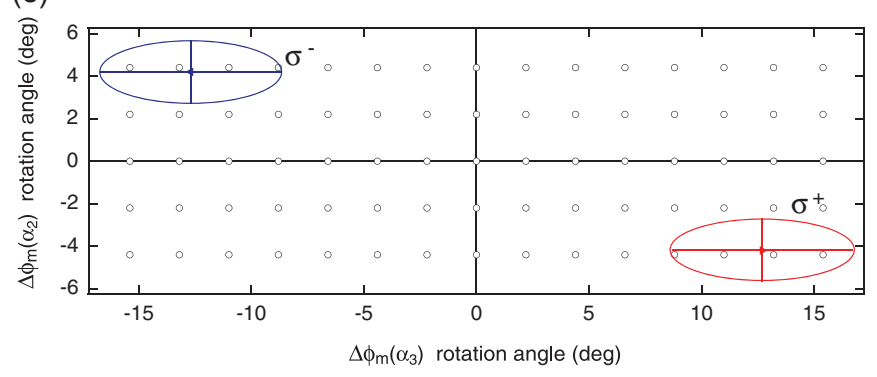

FIG. 4. (Color online) (a)-(d) Asymmetry patterns with the same orientation as in Fig. 3 of (a) $\Delta \alpha_{2}^{-}$, (b) $\Delta \alpha_{2}^{+}$, (c) $\Delta \alpha_{3}^{-}$, and (d) $\Delta \alpha_{3}^{+}$ (for the definition see text). The ticks lie on the azimuth of the node of the corresponding dichroic dipole. The solid triangles indicate the rotation toward the magnetization direction. (e) Rotation angles $\Delta \phi_{m}\left(\alpha_{2}^{ \pm}\right)$vs $\Delta \phi_{m}\left(\alpha_{3}^{ \pm}\right)$. For a given light polarization the $L_{2} M M$ and the $L_{3} M M$ electrons rotate in opposite directions. The ellipses represent the error bars. The open circles are the rotation angles as expected from quantized angular momenta $n \hbar$.

a large, opposite angular momentum compared to that of the photons. It gives insight into the many-body physics upon a $2 p_{1 / 2} \rightarrow 2 p_{3 / 2}$ core hole relaxation and implies a strong angular momentum rearrangement. In particular, the results emphasize that the information on the angular momentum of an electron source wave may not only be accessed by a forward scattering peak rotation, ${ }^{13}$ but, for magnetic systems, also by the precise measurement of the source-wave-dependent circular magnetic dichroism.

In summary, it has been shown that resonant x-ray photoelectron diffraction is suitable to extract the atomic and magnetic structure of surfaces and interfaces. Furthermore, it is demonstrated that the method directly accesses the angular momenta of the emitted electrons.

This paper bases on the arXiv submission 1103.4280. Fruitful discussions with J. Osterwalder and the support of the Swiss National Science Foundation are gratefully acknowledged. The experiments have been performed at the Swiss Light Source. 
*greber@physik.uzh.ch

${ }^{1}$ M. A. Van Hove, Surf. Interface Anal. 28, 36 (1999).

${ }^{2}$ C. S. Fadley, J. Electron Spectrosc. Relat. Phenom. 178, 2 (2010).

${ }^{3}$ P. Krüger et al., Phys. Rev. Lett. 100, 055501 (2008).

${ }^{4}$ M. Treier, P. Ruffieux, R. Fasel, F. Nolting, S. Yang, L. Dunsch, and T. Greber, Phys. Rev. B 80, 081403 (2009).

${ }^{5}$ H. Magnan, P. Le Fevre, D. Chandesris, P. Krüger, S. Bourgeois, B. Domenichini, A. Verdini, L. Floreano, and A. Morgante, Phys. Rev. B 81, 085121 (2010).

${ }^{6}$ C. Guillot, Y. Ballu, J. Paignè, J. Lecante, K. P. Jain, P. Thiry, R. Pinchaux, Y. Pètroff, and L. M. Falicov, Phys. Rev. Lett. 39, 1632 (1977).

${ }^{7}$ L. A. Feldkamp and L. C. Davis, Phys. Rev. Lett. 43, 151 (1979).

${ }^{8}$ L. H. Tjeng, C. T. Chen, P. Rudolf, G. Meigs, G. van der Laan, and B. T. Thole, Phys. Rev. B 48, 13378 (1993).

${ }^{9}$ G. van der Laan, Int. J. Mod. Phys. B 8, 641 (1994).

${ }^{10}$ M. Weinelt, A. Nilsson, M. Magnuson, T. Wiell, N. Wassdahl, O. Karis, A. Föhlisch, N. Mårtensson, J. Stöhr, and M. Samant, Phys. Rev. Lett. 78, 967 (1997).

${ }^{11}$ T. Greber, J. Osterwalder, S. Hüfner, and L. Schlapbach, Phys. Rev. B 45, 4540 (1992).

${ }^{12}$ T. Greber, J. Osterwalder, D. Naumovic, A. Stuck, S. Hüfner, and L. Schlapbach, Phys. Rev. Lett. 69, 1947 (1992).

${ }^{13}$ H. Daimon, T. Nakatani, S. Imada, S. Suga, Y. Kagoshima, and T. Miyahara, Jpn. J. Appl. Phys., Part 2 32, L1480 (1993).

${ }^{14}$ H. Daimon, Phys. Rev. Lett. 86, 2034 (2001).

${ }^{15}$ F. Matsui, T. Matsushita, and H. Daimon, J. Electron Spectrosc. Relat. Phenom. 178, 221 (2010).
${ }^{16}$ J. Stöhr and H. Siegmann, Magnetism-From Fundamentals to Nanoscale Dynamics (Springer, Berlin, 2006).

${ }^{17}$ C. Westphal, J. Bansmann, M. Getzlaff, and G. Schönhense, Phys. Rev. Lett. 63, 151 (1989).

${ }^{18}$ A. Chassé, W. Kuch, M. Kotsugi, X. Gao, F. Offi, S. Imada, S. Suga, H. Daimon, and J. Kirschner, Phys. Rev. B 71, 014444 (2005).

${ }^{19}$ U. Flechsig, F. Nolting, A. F. Rodríguez, J. Krempaský, C. Quitmann, T. Schmidt, S. Spielmann, and D. Zimoch, AIP Conf. Proc. 1234, 319 (2010).

${ }^{20}$ T. Okuda et al., Phys. Rev. B 80, 180404 (2009).

${ }^{21}$ C. T. Chen, N. V. Smith, and F. Sette, Phys. Rev. B 43, 6785 (1991).

${ }^{22} \mathrm{~S}$. Hüfner, Photoelectron Spectroscopy: Principles and Applications (Springer, Berlin, 2003).

${ }^{23} \mathrm{M}$. Morscher, Ph. D. Thesis, University of Zürich, 2011.

${ }^{24}$ Off resonance the intensity and the asymmetry is approximately one order of magnitude smaller. Therefore, a measurement of the magnetization direction with the same accuracy is estimated to require three orders of magnitude more beamtime.

${ }^{25}$ M. Magnuson, N. Wassdahl, A. Nilsson, A. Föhlisch, J. Nordgren, and N. Mårtensson, Phys. Rev. B 58, 3677 (1998).

${ }^{26}$ J. Osterwalder, T. Greber, A. Stuck, and L. Schlapbach, Phys. Rev. B 44, 13764 (1991).

${ }^{27}$ J. Wider, F. Baumberger, M. Sambi, R. Gotter, A. Verdini, F. Bruno, D. Cvetko, A. Morgante, T. Greber, and J. Osterwalder, Phys. Rev. Lett. 86, 2337 (2001).

${ }^{28}$ T. Greber, J. Phys. Condens. Matter 13, 10561 (2001).

${ }^{29}$ A. Chassé and P. Rennert, Phys. Rev. B 55, 4120 (1997). 